\title{
Mild form of Guillain-Barré syndrome in a patient with primary Epstein-Barr virus infection
}

\author{
Se Yong Kim ${ }^{1}$ Kang-Won Choe ${ }^{1}$, Sehhoon Park ${ }^{2}$, Doran Yoon ${ }^{2}$, Chan-Young Ock², Seung Wook Hong ${ }^{2}$, \\ and Jung Yeon $\mathrm{Heo}^{3}$
}

${ }^{1}$ Department of Internal Medicine, The Armed Forces Medical Hospital, Seongnam; ${ }^{2}$ Department of Internal Medicine, Seoul National University Hospital, Seoul; ' ${ }^{3}$ Division of Infectious Diseases, Department of Internal Medicine, Chungbuk National University Hospital, Cheongju, Korea
Received: February 6, 2015

Revised : March 18, 2015

Accepted: April 13, 2015

\section{Correspondence to}

Jung Yeon Heo, M.D.

Division of Infectious Diseases,

Department of Internal Medicine, Chungbuk National University

Hospital, 776 1sunhwan-ro,

Heungdeok-gu, Cheongju 28644, Korea

Tel: $+82-43-269-7634$

Fax: +82-43-273-3252

E-mail:jyeon78@naver.com
To the Editor,

The majority of primary Epstein-Barr virus (EBV) infections occur in childhood or adolescence and are typically asymptomatic or nonspecific illnesses. Over $95 \%$ of adults have EBV seroprevalence [1]. Primary EBV infection in adolescents or young adults can result in infectious mononucleosis. Infectious mononucleosis is a clinical syndrome that is most commonly associated with primary EBV infection and characterized by a triad of fever, pharyngitis, and lymphadenopathy. Most patients with infectious mononucleosis spontaneously resolve without complications. However, $0.37 \%$ to $7.3 \%$ of patients may have neurologic complications such as meningoencephalitis and meningitis [2]. Guillain-Barré syndrome (GBS) is a rare neurologic complication that can occur in patients with infectious mononucleosis. There have been only a few case reports of infectious mononucleosis-associated GBS where severe clinical manifestations, complications, or poor outcomes, such as increased mortality, or sequelae have occurred.

We recently experienced a mild form of GBS in a 25-year-old patient who was diagnosed with infectious mononucleosis. This case was different from the severe form of GBS presented in previous reports, and therefore, we describe this case with a document review.
A previously healthy 25-year-old man was admitted to the hospital with a 7-day history of fever, chills, and sore throat. Initial physical examination revealed exudative tonsillar hypertrophy and pharyngeal injection with an approximately $2 \mathrm{~cm}$ non-tender palpable lymph node. Physical examination of the chest and abdomen was unremarkable and within normal limits. Skin lesions, such as rash, were not observed. Neurological examination for motor and sensory systems was unremarkable. The patient's vital signs at admission were body temperature of $38.2^{\circ} \mathrm{C}$, blood pressure of 136/68 $\mathrm{mmHg}$, heart rate of 86 beats per minute, and respiratory rate of 18 breaths per minute. Complete blood count showed a white blood cell count of $7,460 / \mu \mathrm{L}$, hemoglobin concentration of $13.4 \mathrm{~g} / \mathrm{dL}$, and a platelet count of $80 \times 10^{3} / \mu \mathrm{L}$ with $22 \%$ neutrophils, 66\% lymphocytes, and $12 \%$ monocytes. Chemistry demonstrated an elevated C-reactive protein concentration of $9.56 \mathrm{mg} / \mathrm{dL}$, increased aspartate aminotransferase levels of $205 \mathrm{IU} / \mathrm{L}$, and alanine transaminase levels of $216 \mathrm{IU} / \mathrm{L}$. Atypical lymphocytosis was reported on a peripheral blood smear. Serum antibodies against hepatitis A, B, and $\mathrm{C}$, and human immunodeficiency virus were negative. However, the heterophile antibody test was positive; hence, we presumed infectious mononucleosis 
caused by primary EBV infection and started conservative treatments.

Enzyme immunoassay results for EBV-specific antigens were as follows: Epstein-Barr nuclear antigen (EBNA) immunoglobulin G (IgG) of 2.0 (normal range, < 8.o), EBV viral capsid antigen (VCA) IgM of 3.47 (normal range, $<0.91$ ), and IgG of 1.15 (normal range, < 0.91). Serum antibody titers for cytomegalovirus were $\operatorname{IgM}$ of 0.57 (non-reactive, < 0.85 ) and IgG of 94.2 (non-reactive, 6.o AU/ $\mathrm{mL})$. Group A streptococci from throat swabs were not isolated. The patient was finally diagnosed with infectious mononucleosis caused by primary EBV infection. His initial symptoms of fever and sore throat gradually improved by only conservative treatment; therefore, he was supposed to be discharged from the hospital. However, on day 7 , he complained about neurologic symptoms of numbness and paresthesia localized to hands and feet bilaterally. Neurologic symptoms progressed gradually into bilateral upper and lower extremities. The motor power of his extremities was estimated as grade 4 , and deep tendon reflexes were decreased bilaterally $1+$. Therefore, lumbar puncture was performed. The findings of the cerebrospinal fluid (CSF) analysis were a leukocyte count of 4 cells $/ \mathrm{mm}^{3}$, erythrocyte count of o cells/ $\mathrm{mm}^{3}$, glucose concentration of $55 \mathrm{mg} / \mathrm{dL}$, and protein levels of $102 \mathrm{mg} / \mathrm{dL}$, suggesting albuminocytologic dissociation. Brain magnetic resonance imaging was normal.

The patient was diagnosed with GBS, based on clinical symptoms and CSF analysis. A nerve conduction study showed prolonged terminal latency, delayed F-wave response, and decreased compound muscle action potential and sensory nerve action potential in the bilateral extremities. This was a pattern consistent with sensor-motor polyneuropathy. Although the patient was considered for treatment with intravenous immunoglobulin and plasmapheresis, his vital signs (assessed through respiratory and cardiac monitoring) were stable, and progressive motor weakness (estimated grade 3 to 4 ) of the limbs did not develop. After only conservative treatments, the patient fully regained motor and sensory functions without sequelae. He was discharged from hospital on day 30.

This report illustrates a case of a young adult with infectious mononucleosis caused by primary EBV infection who developed GBS with neurologic complications, but did not progress to a severe course (with quadriplegia or respiratory failure) of the disease. The patient recov- ered without sequelae by only receiving supportive care. Although neurologic complications in patients with infectious mononucleosis can occur, most cases have a benign outcome [2]. Nevertheless, there can be severe sequelae such as death or disability in some patients with infectious mononucleosis associated with neurologic complications.

A few cases reports of GBS after EBV infection date back to the 1940s. All previous case reports on GBS associated with primary EBV infection described severe forms of GBS that is involved respiratory failure or quadriplegia [3]. The most common causal infectious agent associated with the mild form of GBS is the EBV, and $5 \%$ to $28 \%$ of all GBS cases due to EBV were described as mild diseases [4]. Thus, it is possible that clinicians overlooked the mild form of GBS in patients with infectious mononucleosis. In cases with initial symptoms of mild distal weakness and paresthesia (such as our case), a diagnosis of GBS could be missed unless neurological examination is precisely performed. After the symptoms of the primary EBV infection had resolved in our patient, numbness and paresthesia in hands and feet occurred. Thereafter, progressive weakness of the limbs was diagnosed as mild (motor grade 3 to 4 ). Nevertheless, we considered neurologic complications, such as GBS, due to the patient's antecedent viral infection history and thorough physical examination, and additional tests (CSF analysis and nerve conduction study) were therefore performed. The present patient with GBS received only conservative treatments and fully recovered without sequelae. Patients with mild weakness need not be treated beyond supportive care. Plasmapheresis or intravenous immunoglobulin, which are well known as effective treatments for patients with GBS, can be used to treat severe cases of GBS, where patients are unable to walk.

This case suggests that the mild form of GBS, which is manifested as mild sensory and motor impairment, can occur in patients with primary EBV infection.

Keywords: Guillain-Barre syndrome; Epstein-Barr virus infections

\section{Conflict of interest}

No potential conflict of interest relevant to this article was reported. 


\section{REFERENCES}

1. Luzuriaga K, Sullivan JL. Infectious mononucleosis. N Engl J Med 2010;362:1993-2000.

2. Tselis AC. Epstein-Barr virus infections of the nervous system. In: Tselis AC, Booss J, eds. Handbook of Clinical Neurology. Amsterdam: Elsevier, 2014:285-305.
3. Kennedy M, Apostolova M. A rare case of infectious mononucleosis complicated by Guillain-Barre syndrome. Neurol Int 2013;5:20-22.

4. Van Koningsveld R, Schmitz PI, Ang CW, et al. Infections and course of disease in mild forms of Guillain-Barre syndrome. Neurology 2002;58:610-614. 